\title{
Forkhead box protein O3a promotes glioma cell resistance to temozolomide by regulating matrix metallopeptidase and $\beta$-catenin
}

\author{
DATONG SUN $^{1 *}$, SHENGHUI YANG $^{1^{*}}$, XUFENG ZHANG ${ }^{2 *}$, SAI LI $^{1}$, \\ LIN WANG $^{1}$, JUNMIN CHEN ${ }^{1}, \mathrm{CHUN} \mathrm{QIU}^{1}$ and $\mathrm{KE} \mathrm{XU}^{3}$
}

\begin{abstract}
${ }^{1}$ Department of Oncology, Hainan Provincial People's Hospital, Haikou, Hainan 571101; ${ }^{2}$ Department of Stomatology, First Affiliated Hospital of Hainan Medical University, Haikou, Hainan 570102; ${ }^{3}$ Clinical Immunology Section, School of Tropical Medicine and Laboratory Medicine, Hainan Medical University, Haikou, Hainan 571199, P.R. China
\end{abstract}

Received June 6, 2020; Accepted December 18, 2020

DOI: $10.3892 / \mathrm{ol} .2021 .12580$

\begin{abstract}
Glioblastoma multiforme (GBM) is the most common type of malignant brain tumor. GBM is currently treated with temozolomide (TMZ), although patients often exhibit resistance to this agent. Although several mechanisms underlying the resistance of GBM to TMZ have been identified, the combination of these mechanisms is not sufficient to fully account for this phenomenon. Our previous study demonstrated that knocking down the Forkhead box protein O3a (FoxO3a) gene, a member of the FoxO subfamily of transcription factors, resulted in glioma cell sensitization to TMZ, accompanied by reduced levels of nuclear $\beta$-catenin. The aim of the present study was to specify how FoxO3a and $\beta$-catenin are implicated in glioma cell TMZ resistance. Using the U87 and U251 parental cell lines (also designated as sensitive cell lines) and corresponding resistant cell lines (U87-TR and U251-TR, generated by repeated TMZ treatments), coupled with a combined knockdown/overexpression strategy, it was revealed that FoxO3a or $\beta$-catenin overexpression in TMZ-treated U87 and U251 cells markedly increased cellular proliferation; co-expression of both FoxO3a and $\beta$-catenin resulted in the highest increase. Knockdown of either FoxO3a or $\beta$-catenin in U87-TR and U251-TR cells led to a significant
\end{abstract}

Correspondence to: Dr Chun Qiu, Department of Oncology, Hainan Provincial People's Hospital, 19 Xiuhua Road, Haikou, Hainan 571101, P.R. China

E-mail: 13976242127@139.com

Professor Ke Xu, Clinical Immunology Section, School of Tropical Medicine and Laboratory Medicine, Hainan Medical University, 3 School Road, Haikou, Hainan 571199, P.R. China

E-mail: xuke1983amze@163.com

*Contributed equally

Key words: Forkhead box protein O3, $\beta$-catenin, matrix metallopeptidase 9, U251, U87 decrease in cell viability, which was rescued by the re-expression of FoxO3a in FoxO3a-knockdown cells. Subsequent experiments demonstrated that, in U87-TR and U251-TR cells, FoxO3a knockdown significantly reduced the protein levels of matrix metallopeptidase (MMP)9, while overexpression of FoxO3a in U87 and U251 cells enhanced the nuclear accumulation of $\beta$-catenin, concomitantly with an increase in MMP9 levels. Furthermore, MMP9 knockdown markedly reduced the levels of nuclear $\beta$-catenin. Collectively, the findings of the present study suggest that FoxO3a may regulate the nuclear accumulation of $\beta$-catenin by modulating MMP9 expression, thereby rendering glioblastoma cells resistant to TMZ, and may provide unique molecular insights into the mechanisms underlying the development of TMZ resistance in GBM.

\section{Introduction}

Glioblastoma multiforme (GBM) is the most common and most lethal type of primary malignant brain tumor (1). Initially, GBM responds favorably to intensive multimodal treatment comprising surgical resection combined with radiation and chemotherapy; however, patients often experience rapid recurrence, due to the highly chemoresistant tumors $(2,3)$, with a poor prognosis (4) and a median survival time of $<15$ months (5). Temozolomide (TMZ) is an alkylating agent that is currently used as a first-line chemotherapeutic agent against GBM (6-8), although chemoresistance to TMZ has been identified as a major cause of pretreatment failure. Therefore, an improved understanding of the mechanisms through which GBM obtains resistance to TMZ may aid the development of improved treatment methods. Multiple studies have been conducted to determine the mechanisms underlying TMZ resistance, the majority of which focus on O6-methylguanine-DNA methyltransferase (MGMT), which mediates TMZ-induced cytotoxicity. However, MGMT alone does not fully account for the chemoresistance of GBM to TMZ (9-11). This has prompted the investigation of other genes implicated in $\mathrm{TMZ}$ resistance. On the basis of our previous work (12), the present study focused on the involvement of Forkhead box protein $\mathrm{O} 3 \mathrm{a}$ (FoxO3a)/ $\beta$-catenin in TMZ resistance of GBM. 
$\beta$-catenin, a member of the catenin protein family, is a subunit of the cadherin protein complex that serves as a fundamental component of the Wnt signaling pathway $(13,14)$. Numerous studies have demonstrated that $\beta$-catenin is implicated in GBM development and progression. For example, $\beta$-catenin has been positively correlated with the grade of glial neoplasms $(15,16)$ and has been identified as a marker of poor prognosis in patients with glial neoplasms (17). Additionally, the nuclear accumulation of $\beta$-catenin has been associated with a poorer cancer prognosis compared with $\beta$-catenin localization at the cell membrane (18-20). $\beta$-catenin not only serves an important role in cancer, but is also implicated in chemoresistance. Nuclear $\beta$-catenin mediates the continuous activation of the $\mathrm{Wnt} / \beta$-catenin pathway, and confers doxorubicin resistance on neuroblastoma (5). $\beta$-catenin activation by glycogen synthase kinase-3 inhibitor induces chemoresistance to interferon- $\alpha / 5$-fluorouracil combination therapy in hepatocellular carcinoma (21). More importantly, the downregulation of $\beta$-catenin in melanoma cell lines significantly increased the effectiveness of TMZ, cisplatin and doxorubicin, and the inhibition of $\beta$-catenin chemosensitized resistant GBM cells (5).

FoxO3a, a Forkhead box O (FoxO) protein of the Forkhead family, plays a key role in the regulation of cellular differentiation, proliferation and survival (22). Although FoxO3a has been defined as a ubiquitous tumor suppressor, as it induces apoptosis (23), emerging evidence indicates that FoxO3a is strongly associated with poor clinical outcome in specific types of cancer $(24,25)$. For example, FoxO3a enhanced cellular proliferation and invasiveness in specific glioma cell types (26), and has been closely implicated in multidrug resistance in a limited number of cancers $(27,28)$. In agreement with these findings, one of our previous studies indicated that FoxO3a depletion may sensitize glioma cells to TMZ, along with the inhibition of $\beta$-catenin nuclear entry (12). However, whether re-expression of FoxO3a reverses the TMZ-mediated impairment in cell viability, and how FoxO3a regulates the nuclear entry of $\beta$-catenin, remain to be elucidated. The aim of the present study was to thoroughly investigate the specific roles of FoxO3a and $\beta$-catenin in glioma cell TMZ resistance and elucidate the possible underlying mechanisms.

\section{Materials and methods}

Cell lines and culture. The U251 glioma cell line was obtained from the Cell Bank of the Chinese Academy of Sciences (Shanghai, China), and U87 cells (cat. no. HTB-14; glioblastoma of unknown origin) were obtained from the American Type Culture Collection. Both cell lines were cultured in Dulbecco's modified Eagle's medium (Gibco; Thermo Fisher Scientific, Inc.) containing $2 \mathrm{mM}$ glutamine, $10 \%$ fetal calf serum, $100 \mathrm{U} / \mathrm{ml}$ penicillin and $100 \mu \mathrm{g} / \mathrm{ml}$ streptomycin (all from Sigma-Aldrich; Merck $\mathrm{KGaA}$ ), and maintained at $37^{\circ} \mathrm{C}$ in a $5 \% \mathrm{CO}_{2}$ incubator.

Generation of TMZ-resistant GBM cells. To generate TMZ-resistant colonies, parental U251 and U87 cells were exposed to TMZ for 3 weeks. Briefly, the cell lines we initially cultured in six-well plates and allowed to adhere overnight at $37^{\circ} \mathrm{C}$. Treatment with $400 \mu \mathrm{M}$ TMZ was repeated every $24 \mathrm{~h}$ for 5 consecutive days, and the cells were then exposed to fresh TMZ every 3 days for a total of 3 weeks. At the end of the treatment period, a small population of cells had survived and propagated. The surviving colonies were selected and established as TMZ-resistant U251 (U251-TR) and U87 (U87-TR) cell lines.

Establishment of stable cell lines. DNA oligos encoding human FoxO3a short hairpin (sh)RNA (5'-GCATGTTCA ATGGGAGCTTGGA-3') were designed using BLOCK-iT RNAi Designer (Invitrogen; Thermo Fisher Scientific, Inc.), and synthesized and cloned into the pHY-LV-KD1.1 vector (HanYin Biotech) to generate pHY-FoxO3a-KD2, as previously described (26). A vector expressing shRNA against an irrelevant sequence (5'-TGGTTTACATGTCGACTAA-3') was used as the negative control (shRNA-NC). Full-length human FoxO3a cDNA was purchased from Open Biosystems (Horizon Discovery Ltd.) and sub-cloned into the PHY-LV-OE1.6 vector (HanYin Biotech). The construct expressing FoxO3a was designated pHY-FoxO3a-OE. The Trans-Lentiviral Packaging System and the Vira Power Lentiviral Expression System (Invitrogen; Thermo Fisher Scientific, Inc.) were used to produce shRNA and overexpression lentiviruses, respectively. In addition, shRNAs targeting matrix metallopeptidase (MMP)9 (MMP9-sh1, 5'-CGGCAATGCTGA TGGGAAA-3'; MMP9-sh2, 5'-CTTCCAGTACCGAGA GAAA-3'; and MMP9-sh3, 5'-GGCAGCTGGCAGAGGAAT A-3') and $\beta$-catenin (5'-GCATAACCTTTCCCATCATCG-3') were constructed as aforementioned. These constructs were co-transfected with packaging plasmids into $293 \mathrm{~T}$ cells using Lipofectamine ${ }^{\circledR} 2000$ (Invitrogen; Thermo Fisher Scientific, Inc.), according to the manufacturer's instructions; the viral particles were harvested $48 \mathrm{~h}$ later. Upon lentivirus-mediated transduction, stable cell lines were generated via puromycin selection ( $2 \mu \mathrm{g} / \mathrm{ml}$; Sigma-Aldrich; Merck KGaA).

Cell viability assay. Cell viability was determined using the Cell Counting Kit 8 (CCK-8) assay. Cells were seeded into 96-well plates at a density of $3 \times 10^{3}$ cells/well. After overnight incubation at $37^{\circ} \mathrm{C}$, the cells were transduced with lentivirus and then treated with various concentrations of TMZ (range, 200-2,000 $\mu \mathrm{M}$ ) for 1 to 5 days. After a 2-h incubation with $10 \mu \mathrm{l}$ CCK-8 solution (Dojindo Molecular Technologies, Inc.), cell viability was assessed at OD450 nm using a microplate reader (BioTek Instruments, Inc.). The survival rate of untreated cells was set at $100 \%$ and used to calculate the half-maximal inhibitory concentration $\left(\mathrm{IC}_{50}\right)$. Each experiment was conducted in triplicate.

Reverse transcription-quantitative PCR (RT-qPCR) analysis. Total RNA was extracted from the TMZ-resistant and GBM parental cell lines using RNeasy Plus Mini Kit (Qiagen, Inc.), according to the manufacturer's protocol. cDNA was prepared with $1 \mu \mathrm{g}$ total RNA from each sample using SuperScript ${ }^{\circledR}$ VILO $^{\text {TM }}$ cDNA Master Mix (Thermo Fisher Scientific, Inc.); 6 ng cDNA was then used for qPCR analysis in a final reaction volume of $20 \mu \mathrm{l}$. Samples were analyzed in triplicate, and statistical analysis was performed using ANOVA. The qPCR conditions included initial denaturation at $95^{\circ} \mathrm{C}$ for $5 \mathrm{~min}$, denaturation at $95^{\circ} \mathrm{C}$ for $15 \mathrm{sec}$ annealing and extension at $60^{\circ} \mathrm{C}$ for $1 \mathrm{~min}$ with 40 cycles. 
The following primers were used: FoxO3a forward, 5'-AAGCCAGCTACCTTCTCTTCCA-3' and reverse, 5'-GTG GCAAGTCAGTCCGAACTGA-3'; GAPDH forward, 5'-CGG AGTCAACGGATTTGGTCGTAT-3' and reverse, 5'-AGC CTTCTCCATGGTGGTGAAGAC- 3 '; and $\beta$-catenin forward, 5'-CCTCCAGGTGACAGCAATCAG-3' and reverse, 5'-GCC CTCTCAGCAACTCTACAG-3'.

Western blotting. RIPA lysis buffer (cat. no. P0013C; Beyotime Institute of Biotechnology) was used to extract total cellular protein, and the BCA kit (cat. no. P0009; Beyotime Institute of Biotechnology) was used to determine the protein concentration. The proteins in the SW20 and LOVO cell lysates were separated by $10 \%$ SDS-page with $50 \mu \mathrm{g}$ total protein loaded per lane. The proteins were then transferred to nitrocellulose membranes and the membranes were incubated with the appropriate primary antibodies. The primary antibodies were the following: MMP9 (cat. no. 3852; Cell Signaling Technology, Inc., dilution 1:1,000), $\beta$-actin (cat. no. ab8227; Abcam, dilution 1:1,000), $\beta$-catenin (cat. no. 8480; Cell Signaling Technology, Inc., dilution 1:1,000) and Histone H3 (cat. no. ab1791; Abcam, dilution 1:1,000). The secondary antibody was goat anti-rabbit (cat. no. ab150077; Abcam, dilution 1:1,000). The primary antibodies were incubated overnight at $4{ }^{\circ} \mathrm{C}$ and the secondary antibody was incubated for $1 \mathrm{~h}$ at room temperature. The BeyoECL Plus kit (cat. no. P0018S, Beyotime Institute of Biotechnology) was used for the chromogenic protein bands with Beckman Coulter Immunoassay System (UniCel DxI 800; Beckman Coulter). Nuclear and cytoplasmic fractions of the total protein were separated using NE-PER Nuclear and Cytoplasmic Extraction Reagent (Thermo Fisher Scientific, Inc.) and then subjected to western blotting.

Dataset collection and analysis. Survival analysis was performed with Kaplan-Meier plots, and The Cancer Genome Atlas (TCGA) data were analyzed using $\mathrm{R}$ version 3.6.3, along with the 'edgr' package (https://bioconductor.org/packages/release/bioc/html/edgeR.html).

Statistical analysis. Graphically presented data represent the mean $\pm \mathrm{SD}$ of three independent experiments. The differences among groups were determined by one-way ANOVA with Tukey's HSD as post hoc test, and $\mathrm{P}<0.05$ was considered to indicate a statistically significant difference.

\section{Results}

FoxO3a and $\beta$-catenin confer TMZ resistance on glioma cells. Initially, TMZ-resistant cell lines (U87-TR and U251-TR) were developed from two parental tumor cell lines (designated as TMZ-sensitive), both of which are typical glioma cell lines (U87 and U251). The $\mathrm{IC}_{50}$ values 205 and $733 \mu \mathrm{m}$ were selected for subsequent experiments using U87 and U87-TR cells, and 260.1 and $817.6 \mu \mathrm{m}$ for those with U251 and U251-TR cells, as presented in a previous study (12). In order to investigate the functional contributions of FoxO3a and $\beta$-catenin to glioma cell TMZ resistance, a combination of knockdown and overexpression analyses were conducted using both TMZ-sensitive cells and their corresponding resistant counterparts. Specifically, cells stably overexpressing
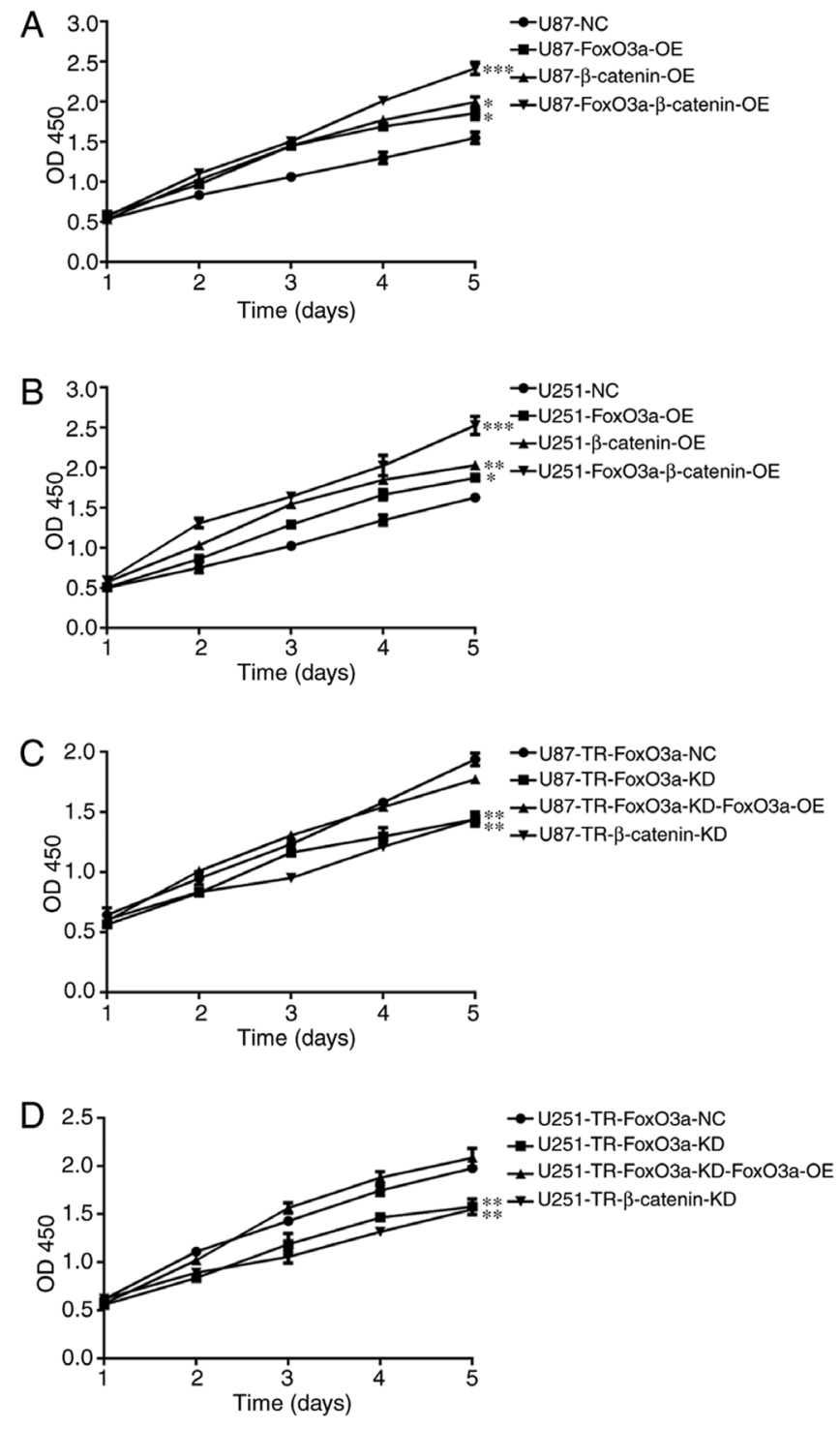

Figure 1. Effects of FoxO3a and $\beta$-catenin on the viability of U87 and U251 parental cells and their corresponding resistant counterparts (U87-TR and U251-TR) following TMZ treatment. Dynamic changes in the viability of (A) U87 and (B) U251 cells after the overexpression of FoxO3a or $\beta$-catenin, or both, for 5 consecutive days (other groups vs the U87- or U251-NC group). Dynamic changes in the viability of (C) U87-TR and (D) U87-TR cells after knockdown of either FoxO3a or $\beta$-catenin, or a combination of FoxO3a knockdown and overexpression for 5 consecutive days (other groups vs. the U87-TR-FoxO3a-NC or U251-TR-FoxO3a-NC group). "P<0.05, ${ }^{* *} \mathrm{P}<0.01$ and ${ }^{* * *} \mathrm{P}<0.001$. FoxO3a, Forkhead box protein O3a; TMZ, temozolomide; $\mathrm{NC}$, negative control; OE, overexpression; KD, knockdown.

FoxO3a or $\beta$-catenin (mediated by lentiviral infection) were generated from the sensitive cell lines, while stably silenced FoxO3a or $\beta$-catenin cells were generated from the resistant cell lines; ectopic overexpression of FoxO3a was performed in the context of FoxO3a knockdown or $\beta$-catenin overexpression. These overexpression and silenced cell lines were then treated with relevant concentrations of TMZ for 5 consecutive days. Cell viability was assessed daily using the CCK-8 assay. As shown in Fig. 1A, the overexpression of FoxO3a markedly increased U87 cell proliferation when compared with U87-NC (U87 negative control). A similar trend was observed in U251 cells (Fig. 1B). It appears that either FoxO3a or $\beta$-catenin overexpression alone resulted in 
A

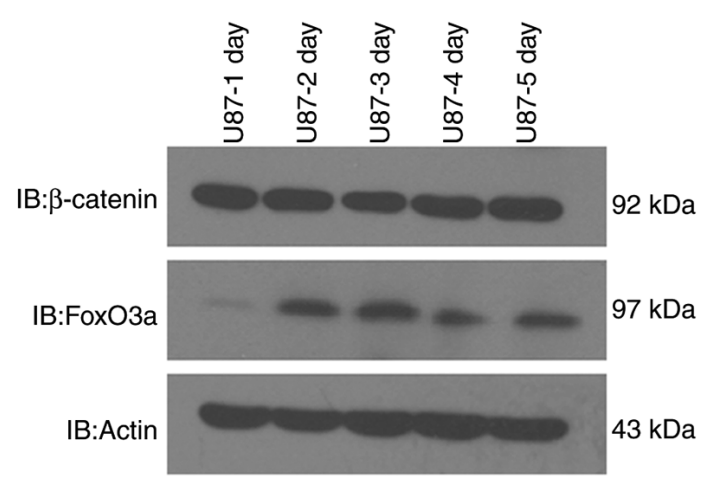

C

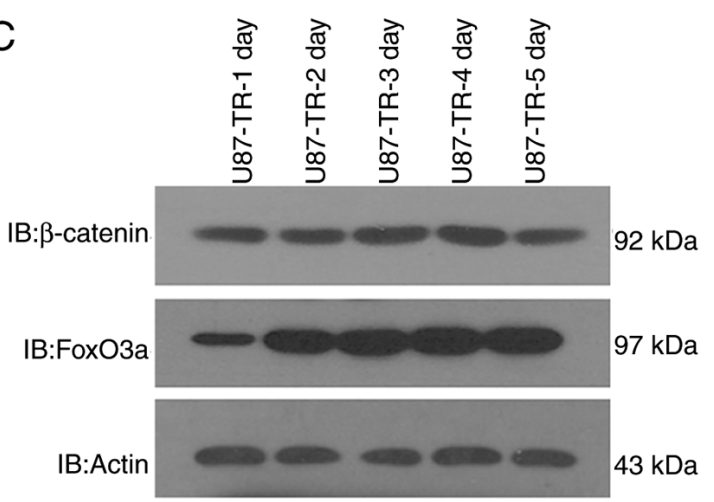

E

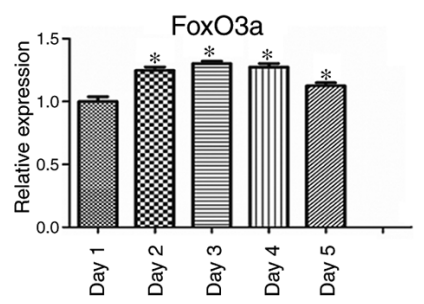

G

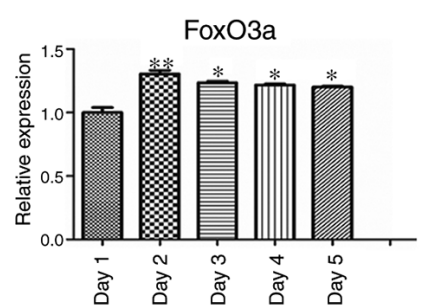

U87

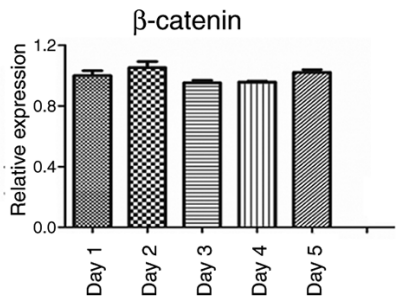

U87-TR

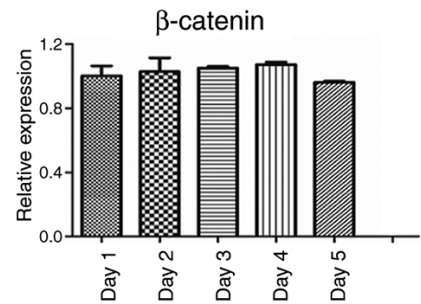

B

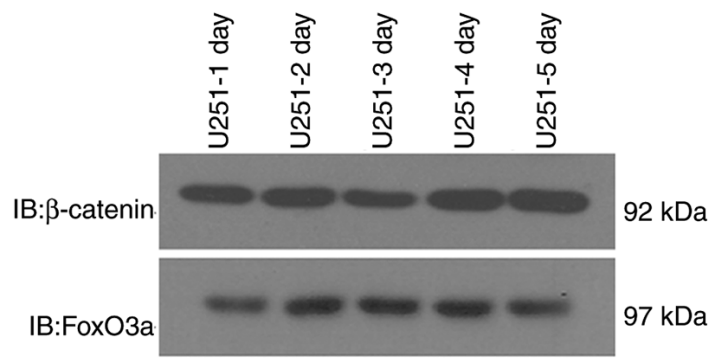

IB:Actin
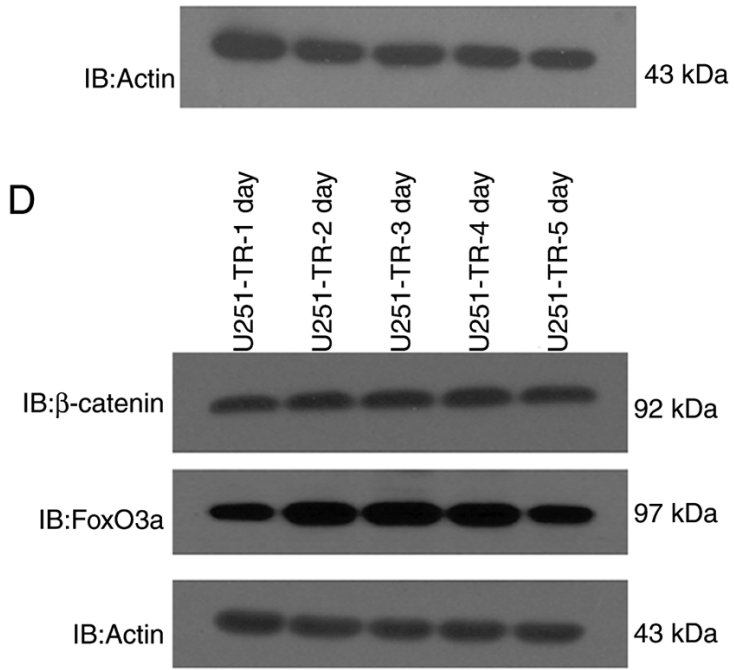

F

U251
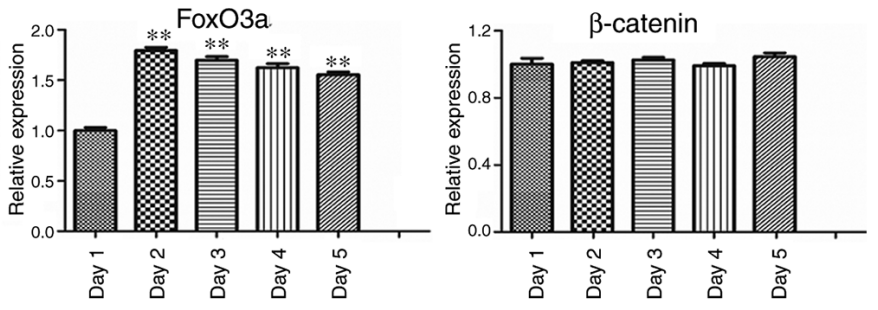

$\mathrm{H}$

U251-TR
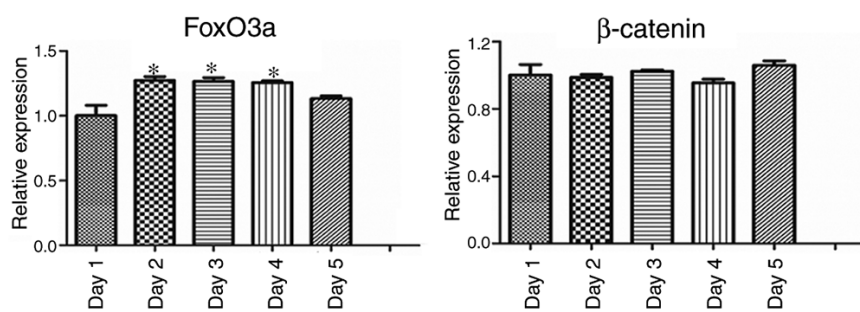

Figure 2. Dynamic changes in FoxO3a and $\beta$-catenin expression over time. (A and B) Protein levels of FoxO3a and $\beta$-catenin in U87 and U251 cells treated with TMZ for 5 consecutive days (other days vs. day 1). (C and D) Protein levels of FoxO3a and $\beta$-catenin in U87-TR and U251-TR cells treated with TMZ for 5 consecutive days (other days vs day 1). (E and F) mRNA levels of FoxO3a and $\beta$-catenin in U87 and U251 cells with TMZ treatment for 5 consecutive days (other days vs. day 1). ( $\mathrm{G}$ and $\mathrm{H}$ ) mRNA levels of FoxO3a and $\beta$-catenin in U87-TR and U251-TR cells treated with TMZ for 5 consecutive days (other days vs. day 1). mRNA data represent the mean $\pm \mathrm{SD}$ of three independent experiments. ${ }^{*} \mathrm{P}<0.05$ and ${ }^{* *} \mathrm{P}<0.01$. FoxO3a, Forkhead box protein $\mathrm{O} 3 \mathrm{a}$; TMZ, temozolomide; TR, temozolomide-resistant.

a comparable increase in U87 cell proliferation (Fig. 1A). As predicted, this trend was also observed in U251 cells (Fig. 1B). Notably, co-expression of both FoxO3a and $\beta$-catenin led to the highest increase in proliferation (Fig. 1A and B). Next, the effects of overexpression and knockdown were assessed in TMZ-resistant cells. Since our previous work demonstrated that FoxO3a and $\beta$-catenin were upregulated in resistant cell lines (12) relative to their sensitive counterparts, the roles of FoxO3a and $\beta$-catenin in TMZ resistance were explored in the context of the relevant gene knockdown in the present study. As shown in Fig. 1C, knocking down FoxO3a or $\beta$-catenin in U87-TR cells significantly decreased cellular proliferation, 

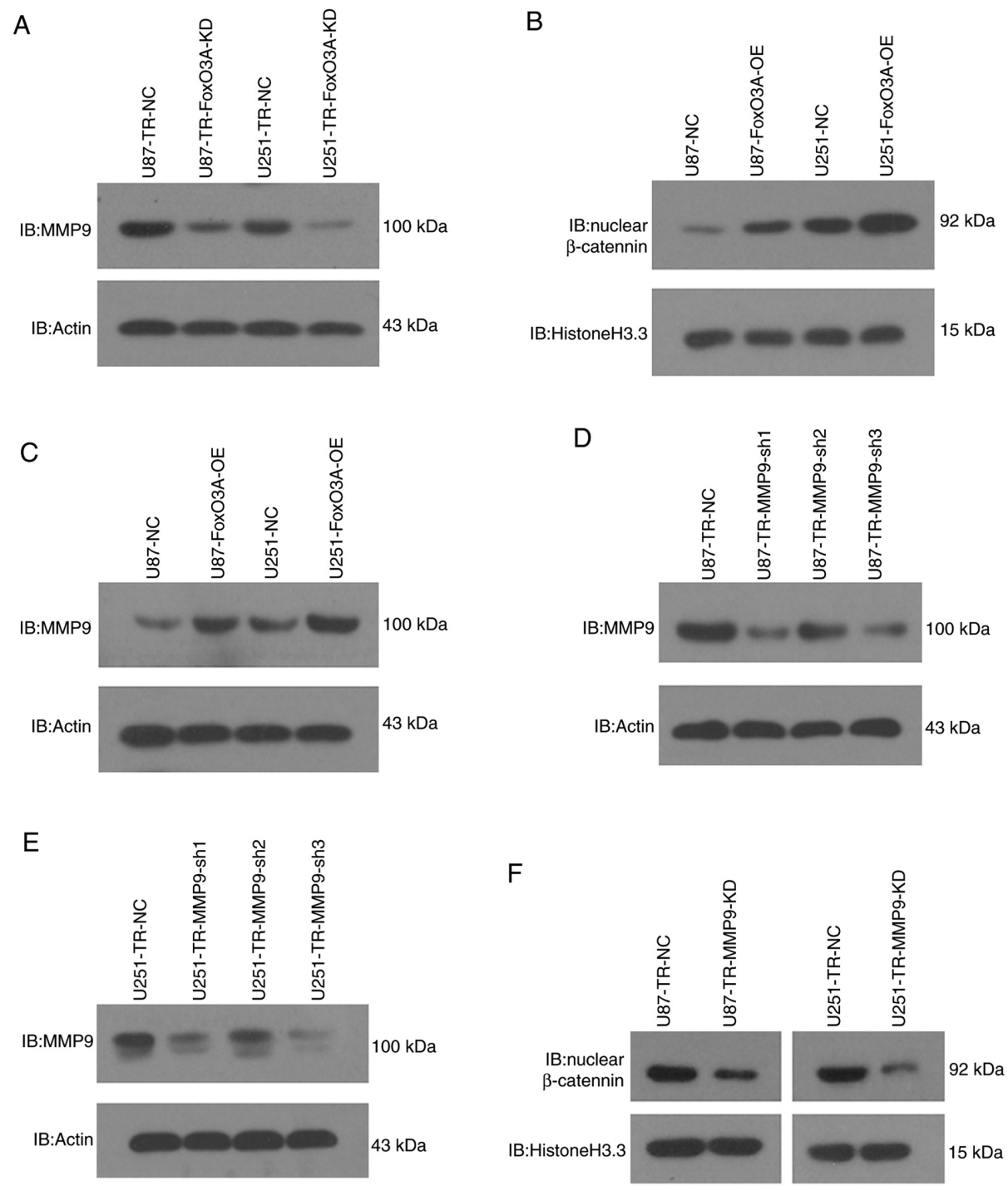

Figure 3. Effect of FoxO3a-induced MMP9 on the nuclear localization of $\beta$-catenin. (A) Protein levels of MMP9 following FoxO3a knockdown in U87-TR and U251-TR cells. (B) Protein levels of nuclear $\beta$-catenin following FoxO3a overexpression in U87 and U251 cells. Histone H3.3 was used as a nuclear marker. (C) Protein levels of MMP9 upon overexpression of FoxO3a in U87 and U251 cells. Selection of an optimal MMP9 shRNA in (D) U87-TR and (E) U251-TR cells. (F) Protein expression levels of nuclear $\beta$-catenin following FoxO3a knockdown in U87-TR and U251-TR cells. FoxO3a, Forkhead box protein O3a; MMP9, matrix metallopeptidase 9; TR, temozolomide-resistant; shRNA, short hairpin RNA; OE, overexpression; KD, knockdown.

which became more apparent over time. Consistent with the observations in U87-TR cells, FoxO3a or $\beta$-catenin knockdown in U251-TR cells also resulted in a marked decrease in proliferation (Fig. 1D). While the depletion of either FoxO3a or $\beta$-catenin resulted in a notable impairment in cell viability, we hypothesized that the re-expression of FoxO3a in knockdown cells may restore TMZ resistance capability. As shown in Fig. 1C and D, the cell viability levels were comparable between the control and FoxO3a-knockdown cells, as well as those overexpressing FoxO3a, indicating that the FoxO3a knockdown-induced reduction in cell viability was reversed by overexpressing FoxO3a. These results (Figs. 1 and S1) demonstrated that FoxO3a and $\beta$-catenin serve key roles in the TMZ-resistant phenotype of glioma cells.

Expression levels of FoxO3a, but not $\beta$-catenin, in glioma cells are altered over time. Although our previous study revealed that elevated FoxO3a and $\beta$-catenin protein expression is associated with glioma cell TMZ resistance (12), the timing and underlying mechanisms were not specified. A key question is whether the levels of $\mathrm{FoxO} 3 \mathrm{a}$ and $\beta$-catenin protein are altered over time; as our previous work merely reflected an endpoint observation, a dynamic change during this process may have been overlooked. In order to answer this question, both TMZ-sensitive and -resistant cell lines were treated with TMZ for 5 consecutive days, and then assessed from days 1-5. Following the appropriate treatments, the protein lysate was subjected to western blot analysis. In sensitive glioma cells, treatment with TMZ increased the levels of FoxO3a protein up to day 2 , which remained largely unchanged between days 2 and 5 . However, $\beta$-catenin protein expression remained unaltered over the entire TMZ treatment period (Fig. 2A and B). FoxO3a protein levels in the resistant glioma cells were also significantly increased after 2 days of TMZ treatment, and remained largely unchanged thereafter. By contrast, there were no significant differences in $\beta$-catenin protein levels over the course of TMZ treatment. Intriguingly, the change in FoxO3a protein level appeared to be more preserved in the resistant 
cell lines, as it increased sharply on day 2, but there were no apparent changes in expression thereafter (Fig. 2C and D). Conversely, a modest decrease in Foxo3a was observed in U87 cells by day 4 , and this trend was partially reversed by day 5 . A similar decrease in Foxo3a expression was observed in U251 cells on day 5 (Fig. 2A and B). Next, changes at the mRNA level were compared with those observed at the protein level. As shown in Fig. 2E-H, FoxO3a mRNA levels were markedly increased in both the sensitive and resistant cell lines, while no significant change in $\beta$-catenin mRNA levels was observed, which is consistent with the corresponding protein expression observations.

FoxO3a regulates $\beta$-catenin nuclear accumulation by modulating MMP9 expression. In our previous study, the depletion of FoxO3a was found to reduce the nuclear localization of $\beta$-catenin with no discernable effect on its overall level (12), suggesting that FoxO3a may lead to $\beta$-catenin nuclear accumulation. However, demonstrating this remains a challenge, as the underlying molecular mechanisms are yet to be elucidated. Nevertheless, the mechanism by which FoxO3a promotes $\beta$-catenin nuclear accumulation was investigated in the present study. FoxO3a knockdown was found to attenuate MMP9 expression and inhibit glioma cell invasiveness (26), and the invasive phenotype regularly coincides with $\beta$-catenin nuclear accumulation. Moreover, another study uncovered a novel molecular event in which MMP9 allows $\beta$-catenin to enter the nucleus (29). Based on these findings, it was hypothesized that FoxO3a may induce $\beta$-catenin nuclear accumulation by regulating MMP9 expression in glioma cells. As shown in Fig. 3A-C, FoxO3a knockdown significantly reduced the protein levels of MMP9, while overexpressing FoxO3a in TMZ-sensitive cells enhanced the nuclear accumulation of $\beta$-catenin, concomitant with an increase in MMP9. In addition, our previous work indicated that depleting FoxO3a resulted in a marked reduction in the protein levels of nuclear $\beta$-catenin, with no change in its overall level (12). Furthermore, lentivirus-mediated MMP9 knockdown was conducted based on the data presented in Fig. 3D and E, and was found to markedly reduced the levels of nuclear $\beta$-catenin (Fig. 3F). These results suggest that FoxO3a regulates $\beta$-catenin nuclear accumulation by modulating MMP9 expression.

FoxO3a and MMP9 expression are clinically relevant in GBM. The coordinated actions of FoxO3a, MMP9 and $\beta$-catenin in cancer cells have already been determined. Therefore, the association between the expression of those three gene and patient survival was assessed. The relevant patient data (mRNA expression and clinicopathological characteristics) were collected from TCGA and preliminarily processed, followed by division into groups according to the stratified median cutoff values. Briefly, the data from 160 cases were collected, of which 104 were male and 56 were female. The age of the patients ranged between 21 and 89 years, with a median age of 59.5 years. There were no significant differences between any of the three genes between the patient groups, which were divided by a primary median survival time (536 days; data not shown). The patients were then further divided into 4 groups based on median cutoff values (median $1=361$ days, and median $2=154.5$ days). In the group with the poorest survival
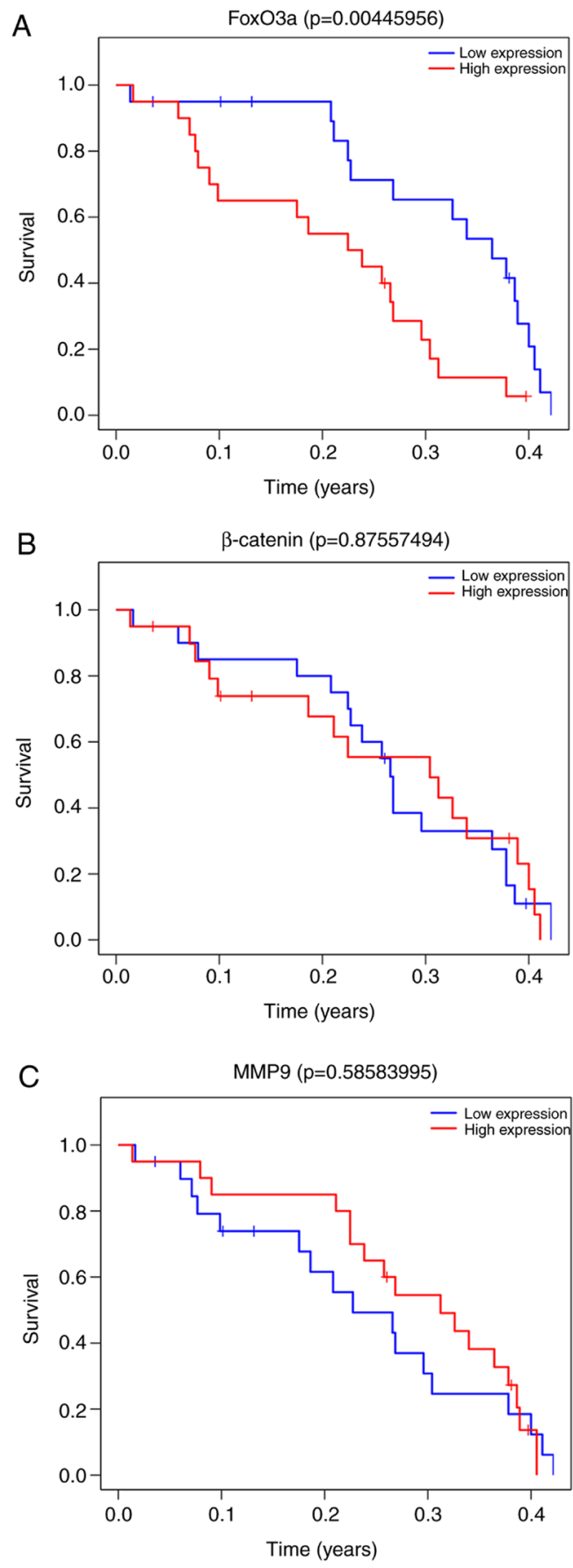

Figure 4. Kaplan-Meier analysis of the association between of FoxO3a, $\beta$-catenin and MMP9 expression and survival of patients with GBM. (A) Comparison between high and low FoxO3a levels in Group 4. (B) Comparison between high and low $\beta$-catenin levels in Group 4. (C) Comparison between high and low MMP9 levels in Group 4. The four groups were determined according to the one primary median and two secondary median survival time points ( 361,536 and 154.5 days). GBM, glioblastoma multiforme; FoxO3a, Forkhead box protein O3a; MMP9, matrix metallopeptidase 9 .

values (Group 4), patients with high levels of FoxO3a expression exhibited shorter survival times compared with those with low FoxO3a levels (Figs. 4A and 5A). This trend was not observed in any of the other groups (Groups 1, 2 and 3), suggesting that low expression levels of FoxO3a play a protective role in the more severe cases. However, similar results were not obtained in association with MMP9 in that group 
(Group 4; Fig. 4C), although the MMP9 expression levels between the groups were significantly different, where they progressively increased until the third group, and then declined in the poorest survival group (Fig. 5C). As predicted, there was no association between $\beta$-catenin level and patient survival, regardless of the associated grouping (Figs. 4B and 5B). These results suggest that the in vitro findings of the present study are consistent with those in clinical samples. However, the findings regarding the changes in the mRNA levels of MMP9 and FoxO3a do not entirely coincide with those at the protein level, which may be attributed to differences in post-translational modification.

\section{Discussion}

A number of studies have revealed that FoxO3a inhibits tumor development and exerts cytostatic and cytotoxic effects, indicative of its tumor-suppressive function (23). However, emerging evidence suggests a correlation between the metastatic functions of FoxO3a and poor cancer prognosis $(24,25)$ which promotes cellular invasiveness, and further supports the oncogenic role of FoxO3a. Moreover, radioresistant glioma cells expressed increased levels of FoxO3a, which, together with nuclear $\beta$-catenin, conferred chemoresistance on colon cancer (30). In agreement with these findings, our previous study demonstrated that glioma-resistant cells exhibit high levels of FoxO3a and $\beta$-catenin, and that FoxO3a induces $\beta$-catenin nuclear accumulation, although the exact mechanisms are yet to be specified (12). For example, when FoxO3a protein levels increase, it is unclear whether the nuclear protein levels of $\beta$-catenin are altered over time, or whether these findings simply represent an endpoint observation. To investigate these questions, cell lines with stable overexpression and knockdown of specific target genes were generated, and a combined knockdown and overexpression strategy was employed. An increase in FoxO3a protein level was present on day 2 , while no change was observed in the overall protein level of $\beta$-catenin. It is conceivable that the increased protein levels FoxO3a were attributed to a corresponding rise in mRNA level in response to TMZ treatment. As predicted, FoxO3a mRNA levels were also increased on day 2 , and remained unchanged between days 2 and 5 , whereas the $\beta$-catenin mRNA level remained unchanged over time. In addition, our previous study (12) merely showed that depleting FoxO3a sensitized glioma cells to TMZ treatment, as well as decreased the protein levels of nuclear $\beta$-catenin. However, it did not fully investigate the following possibilities: i) Whether the overexpression of FoxO3a, $\beta$-catenin, or both, augments glioma cell resistance to TMZ; and ii) Whether the FoxO3a knockdown-induced attenuation of the TMZ-resistant phenotype is reversed by simply re-introducing FoxO3a. To address these issues, a series of experiments were performed on the established stable cell lines. The results demonstrated that overexpressing FoxO3a or $\beta$-catenin alone augmented glioma cell resistance to TMZ, and that the co-overexpression of these two genes imparted the highest degree of TMZ resistance. Despite this, co-overexpression of FoxO3a and $\beta$-catenin appears not to produce a synergistic effect, as the level of TMZ resistance driven by co-overexpression was comparable to that induced by FoxO3a or $\beta$-catenin alone. Additionally, knocking down either FoxO3a or $\beta$-catenin decreased TMZ resistance, while
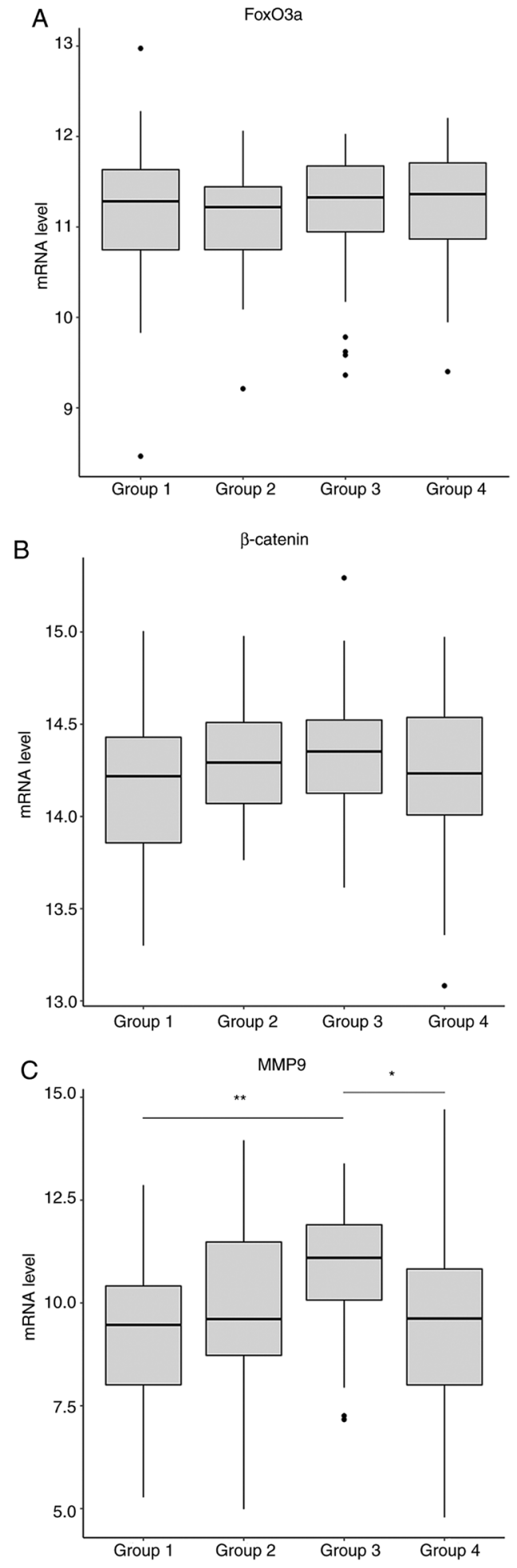

Figure 5. Comparison of FoxO3a, $\beta$-catenin and MMP9 expression between groups. (A) FoxO3a, (B) $\beta$-catenin and (C) MMP9 expression were compared between groups. (C) The four groups were assigned according to the one primary median and two secondary median survival time points $(361,536$ and 154.5 days). ${ }^{*} \mathrm{P}<0.05$ and ${ }^{* *} \mathrm{P}<0.01$ (Kruskall-wallis analysis). 'mRNA level' in the panels represents $\log _{2}$ (gene+1). FoxO3a, Forkhead box protein O3a; MMP9, matrix metallopeptidase 9.

re-introducing FoxO3a (in the context of FoxO3a depletion) restored the TMZ-resistant phenotype, suggesting that FoxO3a is required to maintain TMZ resistance in GBM cells. FoxO3a, together with $\beta$-catenin, potentially underlies the TMZ-resistant 
phenotype of glioma cells. Taken together, these findings support those of our previous study, thus improving our understanding of the roles of FoxO3a and $\beta$-catenin in glioma cell TMZ resistance. However, how FoxO3a promotes $\beta$-catenin accumulation in the nucleus has yet to be fully elucidated.

MMP9 (also known as $92 \mathrm{kDa}$ type IV collagenase, $92 \mathrm{kDa}$ gelatinase and gelatinase B) is a key member of the MMP family involved in the degradation of the extracellular matrix (ECM). MMP9 is implicated in the metastasis and invasion of a variety of cancer types, and acts as a key effector of epithelial-to-mesenchymal transition (EMT). Of note, several recent reports have documented an EMT-like phenotype of glioma cells with increased TMZ resistance capacity (31), suggesting that, in addition to their role in EMT, certain essential EMT genes may also be involved in glioma cell resistance to TMZ. Our previous work $(12,26)$ has demonstrated that FoxO3a promotes glioma cell invasion by upregulating MMP9 protein levels, and that FoxO3a participates in glioma cell TMZ resistance. Furthermore, Dwivedi et al (29) found that MMP9 promotes the entry of $\beta$-catenin into the nucleus. Therefore, we hypothesized that FoxO3a may induce $\beta$-catenin nuclear accumulation by regulating MMP9 expression, thereby augmenting glioma cell resistance to TMZ. Indeed, FoxO3a knockdown significantly reduced the protein expression level of MMP9, whereas overexpression of FoxO3a enhanced the nuclear accumulation of $\beta$-catenin. Moreover, the results of our previous study indicated that depleting FoxO3a results in a marked reduction in the protein levels of nuclear $\beta$-catenin, but does not alter its overall level (12). Furthermore, FoxO3a depletion has previously been reported to reduce MMP9 protein expression (26). These findings suggest that FoxO3a alone is capable of regulating $\beta$-catenin localization and MMP9 expression, but do not conclusively demonstrate that MMP9 plays a critical role in the regulation of nuclear $\beta$-catenin accumulation. Therefore, in the present study, the effects of MMP9 knockdown on the nuclear accumulation of $\beta$-catenin were investigated. Notably, nuclear $\beta$-catenin accumulation was markedly reduced following MMP9 knockdown. These data confirm the previous observations and, together with our previous work, suggest that FoxO3a induces nuclear $\beta$-catenin accumulation primarily by regulating MMP9 expression.

To the best of our knowledge, the results of the present study represent a striking and novel example that links the FoxO3a, MMP9 and $\beta$-catenin genes to glioma cell TMZ resistance, and highlight a novel functional role of MMP9 in TMZ resistance. Such a mechanism appears plausible, as the roles of different genes are often context-dependent (such as the cell type and associated stimulus). Although MMP9 is considered to be an effector of cellular invasiveness and ECM remodeling, an increasing number of studies have indicated alternative roles for MMP9. For example, inhibiting MMP9 prior to the use of cisplatin (which is used to treat ovarian cancer) can reduce cisplatin resistance (32). Moreover, MMP9 augmented tumor cell resistance to natural killer cell-mediated cytotoxicity by cleaving intercellular adhesion molecule 1 (33). In brief, the results of the present study, as well as those of other researchers, support the view that the functions of a particular gene are context-dependent, which may inspire different methods for investigating MMP9.
Another aim of the present study was to investigate the association between FoxO3a, MMP9 and $\beta$-catenin in clinical GBM samples. High levels of FoxO3a mRNA appeared to be associated with the poorest survival outcome, and the difference in MMP9 levels among the stratified groups partly followed suit. Since the localization, but not the expression, of $\beta$-catenin plays a key role in glioma, no correlation was expected between overall $\beta$-catenin mRNA levels and patient survival. As predicted, no significant difference was observed across the patient groups. Although not as solid as expected, these clinical findings remain relevant to the associated in vitro findings.

In conclusion, the results of the present study highlight the FoxO3a/MMP9/ $\beta$-catenin network as a novel regulatory mechanism in glioma cells, which promotes glioma cell resistance to TMZ. However, the more specific molecular signaling events will be investigated in future studies. These findings provide novel insights into the mechanisms of TMZ resistance in glioma cells.

\section{Acknowledgements}

Not applicable.

\section{Funding}

This study was supported by the National Natural Science Foundation of China (grant no. 81660502).

\section{Availability of materials and data}

The datasets used and/or analyzed during the current study are available from the corresponding author on reasonable request.

\section{Authors' contributions}

DS wrote the manuscript and conducted data analysis. SY and XZ performed the experiments. SL, LW and JC collected and analyzed the data and fixed the syntax errors. CQ and KX contributed to the study design and revised the manuscript for important intellectual content. KX and CQ confirm the authenticity of all the raw data presented. All authors have read and approved the final manuscript.

\section{Ethics approval and consent to participate}

Not applicable.

\section{Patient consent for publication}

Not applicable.

\section{Competing interests}

The authors declare that they have no competing interests.

\section{References}

1. Gautam M, Singh S, Aggarwal M, Sharma MK, Dang S and Gabrani R: Glioblastoma multiforme; drug resistance \& combination therapy. Front Anti Cancer Drug Discovery Volume 10: 111,2019 
2. Hegi ME, Diserens AC, Gorlia T, Hamou MF, de Tribolet N, Weller M, Kros JM, Hainfellner JA, Mason W, Mariani L, et al: MGMT gene silencing and benefit from temozolomide in glioblastoma. New Engl J Med 352: 997-1003, 2005.

3. Clarke J, Butowski N and Chang S: Recent advances in therapy for glioblastoma. Arch Neurol 67: 279-283, 2010.

4. Wang Y, Chen L, Bao Z, Li S, You G, Yan W, Shi Z, Liu Y, Yang P, Zhang W, et al: Inhibition of STAT3 reverses alkylator resistance through modulation of the AKT and $\beta$-catenin signaling pathways. Oncol Rep 26: 1173-1180, 2011.

5. Sinnberg T, Menzel M, Ewerth D, Sauer B, Schwarz M, Schaller M, Garbe C and Schittek B: $\beta$-Catenin signaling increases during melanoma progression and promotes tumor cell survival and chemoresistance. PLoS One 6: e23429, 2011

6. Mrugala MM and Chamberlain MC: Mechanisms of disease: Temozolomide and glioblastoma-look to the future. Nat Clin Pract Oncol 5: 476-486, 2008.

7. Agnihotri S, Gajadhar AS, Ternamian C, Gorlia T, Diefes KL, Mischel PS, Kelly J, McGown G, Thorncroft M, Carlson BL, et al: Alkylpurine-DNA-N-Glycosylase confers resistance to temozolomide in xenograft models of glioblastoma multiforme and is associated with poor survival in patients. J Clin Invest 122: 253-266, 2012.

8. Motomura K, Natsume A and Wakabayashi T: Intravenous administration of temozolomide as a useful alternative over ora treatment with temozolomide capsules in patients with gliomas. J Neuroncol 106: 209-211, 2012.

9. Cahill DP, Levine KK, Betensky RA, Codd PJ, Romany CA, Reavie LB, Batchelor TT, Futreal PA, Stratton MR, Curry WT, et al: Loss of the mismatch repair protein MSH6 in human glioblastomas is associated with tumor progression during temozolomide treatment. Clin Cancer Res 13: 2038-2045, 2007.

10. Yip S, Miao J, Cahill DP, Iafrate AJ, Aldape K, Nutt CL and Louis DN: MSH6 mutations arise in glioblastomas during temozolomide therapy and mediate temozolomide resistance. Clin Cancer Res 15: 4622-4629, 2009.

11. Avgeropoulos NG and Batchelor TT: New treatment strategies for malignant gliomas. Oncologist 4: 209-224, 1999.

12. Xu K, Zhang Z, Pei H, Wang H, Li L and Xia Q: FoxO3a induces temozolomide resistance in glioblastoma cells via the regulation of $\beta$-catenin nuclear accumulation. Oncol Rep 37: 2391-2397, 2017.

13. Peifer M, Rauskolb C, Williams M, Riggleman B and Wieschaus E: The segment polarity gene armadillo interacts with the wingless signaling pathway in both embryonic and adult pattern formation. Development 111: 1029-1043, 1991.

14. Noordermeer J, Klingensmith J, Perrimon $\mathrm{N}$ and Nusse $\mathrm{R}$ Dishevelled and armadillo act in the wingless signalling pathway in drosophila. Nature 367: 80-83, 1994.

15. Zhang Z, Chen H, Chen Y and Cheng X: Significance of beta-catenin and Cyclin D1 express in glioma. Xi Bao Yu Fen Zi Mian Yi Xue Za Zhi 25: 1010-1012, 2009 (In Chinese)

16. Liu X, Wang L, Zhao S, Ji X, Luo $Y$ and Ling F: $\beta$-Catenin overexpression in malignant glioma and its role in proliferation and apoptosis in glioblastma cells. Med Oncol 28: 608-614, 2011.

17. Liu C, Tu Y, Sun X, Jiang J, Jin X, Bo X, Li Z, Bian A, Wang X, Liu D, et al: Wnt/Beta-Catenin pathway in human glioma: Expression pattern and clinical/prognostic correlations. Clin Exp Med 11: 105-112, 2011.

18. Pukkila M, Virtaniemi J, Kumpulainen E, Pirinen RT, Johansson RT, Valtonen HJ, Juhola MT and Kosma VM: Nuclear beta catenin expression is related to unfavourable outcome in oropharyngeal and hypopharyngeal squamous cell carcinoma J Clin Pathol 54: 42-47, 2001.

19. Elzagheid A, Buhmeida A, Korkeila E, Collan Y, Syrjänen K and Pyrhönen S: Nuclear beta-catenin expression as a prognostic factor in advanced colorectal carcinoma. World J Gastroenterol 14: 3866-3871, 2008.
20. Huang CL, Liu D, Ishikawa S, Nakashima T, Nakashima N, Yokomise H, Kadota K and Ueno M: Wnt1 overexpression promotes tumour progression in non-small cell lung cancer. Eur J Cancer 44: 2680-2688, 2008

21. Noda T, Nagano H, Takemasa I, Yoshioka S, Murakami M, Wada H, Kobayashi S, Marubashi S, Takeda Y, Dono K, et al: Activation of wnt/beta-catenin signalling pathway induces chemoresistance to interferon-alpha/5-fluorouracil combination therapy for hepatocellular carcinoma. Br J Cancer 100: 1647-1658, 2009.

22. Accili D and Arden KC: FoxOs at the crossroads of cellular metabolism, differentiation, and transformation. Cell 117: 421-426, 2004

23. Myatt SS and Lam EWF: The emerging roles of forkhead box (Fox) proteins in cancer. Nat Rev Cancer 7: 847-859, 2007.

24. Chen J, Gomes AR, Monteiro LJ, Wong SY, Wu LH, $\mathrm{Ng}$ TT, Karadedou CT, Millour J, Ip YC, Cheung YN, et al: Constitutively nuclear FOXO3a localization predicts poor survival and promotes akt phosphorylation in breast cancer. PLoS One 5: e12293, 2010.

25. Storz P, Döppler H, Copland JA, Simpson KJ and Toker A: FOXO3a promotes tumor cell invasion through the induction of matrix metalloproteinases. Mol Cell Biol 29: 4906-4917, 2009.

26. Xu K, Pei H, Zhang Z, Dong S, Fu RJ, Wang WM and Wang H: FoxO3a mediates glioma cell invasion by regulating MMP9 expression. Oncol Rep 36: 3044-3050, 2016.

27. Sun J, Wei H, Yi J, Chen J and Tian B: Human umbilical cord mesenchymal stem cells restore imatinib and doxorubicin sensitivity in drug-resistant chronic myeloid leukemia cells. Int J Clin Exp Med 11: 2142-2147, 2018.

28. Aldonza MBD, Hong JY and Lee SK: Paclitaxel-Resistant cancer cell-derived secretomes elicit ABCB1-associated docetaxel cross-resistance and escape from apoptosis through FOXO3a-driven glycolytic regulation. Exp Mol Med 49: e286, 2017.

29. Dwivedi A, Slater SC and George SJ: MMP-9 and-12 cause $\mathrm{N}$-cadherin shedding and thereby beta-catenin signalling and vascular smooth muscle cell proliferation. Cardiovasc Res 81: 178-186, 2009

30. Tenbaum SP, Ordóñez-Morán P, Puig I, Chicote I, Arqués O, Landolfi S, Fernández Y, Herance JR, Gispert JD, Mendizabal L, et al: $\beta$-Catenin confers resistance to PI3K and AKT inhibitors and subverts FOXO3a to promote metastasis in colon cancer. Nat Med 18: 892, 2012.

31. Kahlert U, Nikkhah G and Maciaczyk J: Epithelial-ToMesenchymal (-like) transition as a relevant molecular event in malignant gliomas. Cancer Lett 331: 131-138, 2013.

32. Laios A, Mohamed BM, Kelly L, Flavin R, Finn S, McEvoy L, Gallagher M, Martin C, Sheils O, Ring M, et al: Pre-Treatment of platinum resistant ovarian cancer cells with an MMP-9/MMP-2 inhibitor prior to cisplatin enhances cytotoxicity as determined by high content screening. Int J Mol Sci 14: 2085-2103, 2013.

33. Fiore E, Fusco C, Romero P and Stamenkovic I: Matrix metalloproteinase 9 (MMP-9/gelatinase B) proteolytically cleaves ICAM-1 and participates in tumor cell resistance to natural killer cell-mediated cytotoxicity. Oncogene 21: 5213-5223, 2002.

This work is licensed under a Creative Commons Attribution-NonCommercial-NoDerivatives 4.0 International (CC BY-NC-ND 4.0) License. 